\title{
End-to-End QoS Provisioning for Real-Time Video Streaming over SP-Driven P2P Networks Using Admission Control
}

\author{
Mubashar Mushtaq and Toufik Ahmed \\ CNRS LaBRI Lab. - University of Bordeaux 1, France \\ \{mushtaq,tad\}@labri.fr
}

\begin{abstract}
Enabling end-to-end Quality of Service (QoS) for realtime video content delivery across heterogeneous P2P networks is challenging but vital for the efficient service provision. In this paper, we present a P2P based admission control mechanism for real-time video streaming driven by Service Provider (SP). SPdriven P2P network is a network where service provider has a comprehensive control over contracted resource utilization. SP assures the coordination and the creation of overlay P2P network and it is responsible for admission control and resource allocation among others. Our proposed QoS provision mechanism employs video traffic descriptor (i.e. traffic specification) for performing admission control. Before accepting a particular P2P video streaming session, the SP controls the access to the network resources by performing policy and admission control. After retrieving the service availability from its traffic repository, SP decides whether to accept or to reject the request. The proposed SP-driven $P 2 P$ admission control mechanism is evaluated using ns2 simulator and the results show that our proposed mechanism allows the provisioning of end-toend QoS for delivered video content over P2P network.
\end{abstract}

Index Terms-SP-Driven P2P Network, QoS, Admission Control, Real-Time Video Streaming

\section{INTRODUCTION}

C URRENT Internet is undergoing through a massive shift from being a simple monolithic data service network to a ubiquitous multi-service network where different stakeholders co-operate for offering value-added services and applications to content consumers (CCs). Content providers (CPs), service providers (SPs), and network providers (NPs) have different roles and requirements for providing services and applications which may dynamically be created and managed. The popular applications that have changed radically the way how consumer interacts with the content are based on peer-to-peer ( $\mathrm{P} 2 \mathrm{P})$ architecture. Today, this $\mathrm{P} 2 \mathrm{P}$ phenomenon is widely adopted for different large-scale content distributions including multimedia streaming, video on demand (VoD), P2P gaming, signaling for IP telephony, P2Pbased IPTV services, etc. Recent measurement studies [1] have shown that traffic generated by P2P applications has started to dominate the bandwidth consumption on Internet access links. In this massive growth of $\mathrm{P} 2 \mathrm{P}$, the abovementioned stakeholders (i.e. SP, CP, NP) are missing opportunities for future revenue growth. On one hand, SPs have no control over the structure of the P2P networks and therefore they can neither control nor manage the associated traffic flows. On the other hand, P2P users just want to retrieve their desired content quickly, with good performance.
However, P2P systems have no knowledge of the underlying network structure and they either have to measure the performance itself or to build its overlay topology more or less agnostic of the underlay. This situation is frustrating to both SPs as well as P2P users. The cooperation between SPs and $\mathrm{P} 2 \mathrm{P}$ users not only improves the network utilization but also allows the SPs to provide better services to their customers [2]. Moreover, such cooperation can be helpful to address the issue of application-layer traffic optimization problem (ALTO) [3]. We believe that SP-driven P2P system will constitute the next generation $\mathrm{P} 2 \mathrm{P}$ system that is willing to provide better QoS performance to CCs whilst enhancing revenue growth for different actors involved in the delivery chain. Explicit communication for cooperative control between $\mathrm{P} 2 \mathrm{P}$ and providers has been recently the subject of a working group called P4P [4] and IETF WG ALTO [3].

In the current practice, SPs deliver the content to consumers using best effort or QoS-enabled infrastructure after aggregating media content from multiple CPs. SPs may not necessarily own or administer any networking infrastructure, but they should interact with network providers through Service Level Agreement (SLA). SLAs describe the characteristics of the offered service and identify the responsibilities of the parties involved in service delivery process. The Service Level Specification (SLS) is the technical characteristics of the service offered in the context of SLA that refers to the provisioning aspects of the service e.g. request, activation and delivery aspects from network perspectives. There is a distinction between customer to provider SLSs and provider-to-provider SLSs. The Client Service Level Agreements/ Client Service Level Specification (cSLA/cSLS) is established between end-customers and service/network providers. The provider service level specification (pSLS) is established between the service and network providers or between network providers. The SLS template includes the following attributes: SLS Identification, Scope of SLS, Flow Identification, Traffic Conformance \& Characteristics, Traffic Excess Treatment, Performance Guarantees to be given to the traffic, etc. This information plays an important role for overall QoS provisioning using admission control.

In this paper, we present a QoS provisioning mechanism for real-time video streaming over SP-driven P2P networks using admission control. The SP-driven P2P framework allows SPs to have a global view of the available bandwidth and maintains a traffic forecast matrix that provides the information regarding allocated and available bandwidth over 
certain network links. SPs further use this information to establish QoS-enabled paths between peers participating in a session for actual content delivery. The admission control mechanism results into efficient utilization of resources by preventing the unauthorized peers from accessing the SPdriven $\mathrm{P} 2 \mathrm{P}$ overlays.

The rest of this paper is organized as follows: section II briefly describes SP-driven P2P framework with some of the related works, section III describes QoS provisioning mechanism, section IV presents performance evaluation of the proposed mechanism, and finally section $\mathrm{V}$ concludes the paper along with some future perspectives.

\section{TARgET ARChiteCtURE \& RELATED WORKS}

SP-driven P2P architecture is shown in Fig. 1 along-with different entities involved in the content distribution chain that include Network Providers (NPs), Service Providers (SPs), Access Network Providers (ANPs), Content Providers (CPs), and Service/Content Consumers (CCs). The e2e QoS provision across $\mathrm{P} 2 \mathrm{P}$ networks is more challenging not only because of the presence of many different paths between CC and potential senders but also due to the dynamicity of P2P architecture, where peers may enter and leave the P2P system without prior notification. However, P2P system provides better scalability for enabling QoS service provisioning in more transparent and cost-effective way. To ensure smooth service delivery in SP-driven P2P system, the service provider has to monitor the network resources regularly (service level monitoring) and/or to perform admission control before service invocation. In our proposed mechanism, the contents are delivered using the e2e aggregated pipes on the basis of traffic descriptor (traffic specification) [6] and traffic forecast matrix that provides the information for available resources on certain network links.

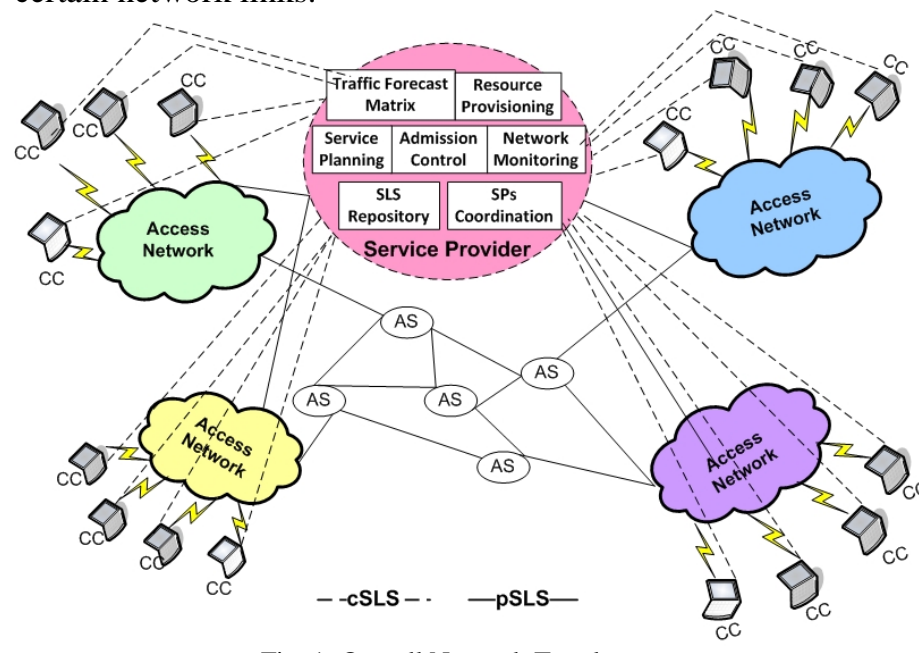

Fig. 1. Overall Network Topology

The proposed SP-driven P2P framework is composed of different entities that have distinguished role in overall service planning and efficient service delivery. Traffic forecast matrix provides a global view of available network resources. Network monitoring is performed to observe the network conditions and further used for the resource provisioning. The SLS repository keeps information of all the service-level negotiations among different entities. The admission control mechanism is implemented to prevent unauthorized access of network by admitting or rejecting traffic flows over certain network links. SPs coordination block is responsible for the negotiations among different SPs through explicit communication for efficient utilization of shared resources.

In SP-driven framework, a SuperNode tracker (SN-tracker) is another important entity that is part of the peers in P2P network and it may be owned by SP or chosen by SP. A SNTracker provides three kinds of services to the P2P applications: (1) Capability: it specifies network provider capabilities such as classes of services, (2) Policy: it specifies, how a network provider would like its network to be utilized, typically, it specifies specific suggestions for P2P applications how and when to use the network resources, and (3) Descriptor that specifies the network status.

The participating peers possess heterogeneous network capabilities for their uplink and downlink. Thus, we focus on a scenario where a single peer receives video content from multiple sender peers. We identify certain network constraints for the admission control by using the traffic specification (TSPEC) model [7]. In our target architecture, we focus on delivering scalable video coding (SVC) composed of different layers or layers [8]. This video encoding scheme helps us to evaluate the delivery of different SVC layers coming from different senders.

The rest of this section discusses some of the existing work carried out in the context of this paper.

$\mathrm{P} 4 \mathrm{P}$ [4] is a framework that provides an extension of the classical P2P framework to address the issues arising with the absence of any control over P2P. It provides a mechanism that allows effective control over network traffic among network providers and certain applications. Such cooperation can be helpful in improving the QoS for application as well as for efficient service provision by the service providers. However, this framework is relatively young and no real mechanism has been proposed by the working group.

Regarding admission control mechanisms for $\mathrm{P} 2 \mathrm{P}$, a distributed rate control mechanism is provided for P2P networks in that is based on the congestion awareness which exploits an optimization model. Each service provider allocates its rate capacity on the basis of the offered price by service consumer. For the multi-source downloading, the rate control algorithm needs to run at both service provider and receiver end to determine the upload and download capacities. This model cannot be adapted to real business model since SLA needs to be established before service invocation.

In [10], an admission control mechanism for media streaming over P2P network is presented that intends to maximize the load allocation problem for the number of concurrent requests for a given set of peers. The proposed algorithm provides resilience for the smooth streaming that is assured by the reliability of peers. The architecture is hybrid since it combines both pure peer-to-peer streaming model and centralized server model in order to take advantages of them.

In [11], an architecture for QoS-aware P2P multimedia network is presented. The QoS is ensured specially by admission control manager that is responsible for assigning the network resources. The architecture utilizes resource reservation protocol (RSVP), that we believe is not scalable. 
In contrast to the other available studies, we focus on the SP-driven P2P networking architecture, where SPs have explicit communications with other entities to efficiently utilize available resources. The SP negotiates with the network provider to maintain its traffic forecast matrix that is further used to allocate the available resources for the streaming services. The potential use of admission control mechanism with P2P networks for service delivery allows the reduction of the network traffic load on the central servers.

\section{Qos PROVISIONING MECHANISM USING ADMISSION CONTROL}

Real-time applications such as video streaming and VoD are highly stringent against fluctuation in network conditions and require certain QoS mechanism to ensure smooth content delivery over $\mathrm{P} 2 \mathrm{P}$ networks. So, the incorporation of an appropriate admission control strategy can be helpful to guarantee an acceptable level of QoS for real-time content delivery over $\mathrm{P} 2 \mathrm{P}$ networks where a single peer seeks to receive video content from multiple sender peers having distinct characteristics. This admission control mechanism is used to control the amount of video traffic injected in each intra-domain and inter-domain links while accepting or rejecting the service requests to ensure QoS. Once transmitted, the video content are received in a particular buffer before the decoding phase. The streaming process is controlled by using a receiver side scheduler which ensures the conformance of the data arrival to the TSPEC model. If the conformance test fails during the content delivery phase, remedial action should be taken either by performing peer/stream switching or by quality adaptation [12]. Toward this end, NP applies a token bucket model for performing admission control of incoming traffic (video streams) from different CPs using TSPEC. TSPEC model is generally applied for QoS negotiations that are based on the pre-defined parameters. SP maintains and updates its traffic matrix to keep track of all used/available resources over the contracted NP domains. Table 1 describes the TSPEC parameters used in our proposed mechanism.

\begin{tabular}{ll} 
TABLE 1. TSPEC PARAMETERS FOR STREAMING APPLICATIONS \\
\hline Acronym & TSPEC Parameter \\
\hline PDR & Peak Data Rate of video layer \\
ADR & Average Data Rate of video layer \\
CDR & Committed Data Rate for allocated bandwidth \\
PBS & Peak Burst Size of video packets \\
$\mathrm{X}_{\mathrm{avg}}$ & Average Packet Size of video packets \\
$\mathrm{X}_{\max }$ & Maximum Packet Size of video packets \\
\hline
\end{tabular}

The proposed streaming mechanism is receiver centric where receiver peer orchestrates the selection of multiple sender peers. However, QoS provisioning is assured by service provider by implementing a SP-driven Admission Control (SP-AC) mechanism. The requested video stream is composed of multiple SVC layers and each sender peer contributes different video quality layers. Receiver peer selects multiple sender peers for different video quality layers and SP implements SP-AC mechanism for QoS provisioning. The overall admission control mechanism is presented in Fig. 2 , where $\mathrm{L}_{1}, \mathrm{~L}_{2}, \mathrm{~L}_{3}$, and $\mathrm{L}_{4}$ correspond to the Base layer and enhancement layers of SVC video. The targeted peers are activated by SP on the basis of respective video layers.

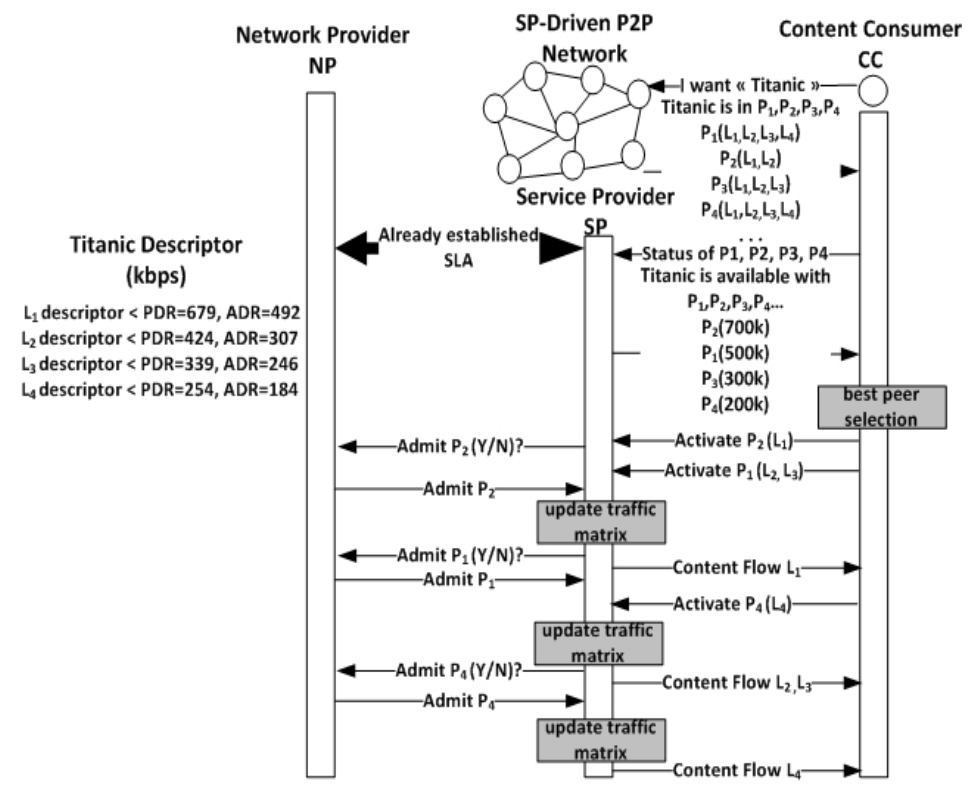

Fig. 2. P2P based SP-AC Mechanism

Since the underlying network topology is based on the P2P architecture, there exist several intermediate nodes involved in the content delivery along the e2e path between sender and receiver peers. Thus, we propose to use a cascade model [5] for SLA/SLS negotiations between SPs and NPs to ensure the overall QoS along the e2e path. The signaling protocol for the TSPEC negotiations is out of scope of this paper.

To provide a smooth video delivery, a receiver side scheduler determines the relative schedules for the each video layer according to their priorities that include transmission time, actual playback time. We consider two sets of parameters to apply admission control mechanism for each video layer. First, the transmission of video packet from the sender peer is done according to relative scheduling time and priority. Second, the video packet should arrive at receiverend before playback deadline (i.e., maximum delay bound).

These proposed set of parameters are used to determine the viability of received video packets. The video packets received after the respective playback deadlines are discarded alongwith the corresponding video packets of upper layers. We presented these set of parameters by $\beta_{k}^{\text {schedule }}=\left\{s_{k}, p_{k}^{\text {playback }}, p_{k}^{\text {transmit }}\right\}$ and $\beta_{k}^{\text {receive }}=\left\{s_{k}, p_{k}^{\text {playback }}, p_{k}^{\text {receive }}\right\}$. Here, $P_{k}^{\text {playback }}, P_{k}^{\text {transmit }}$, and $P_{k}^{\text {receive }}$ represent relative playback time, transmission time, and receiving time of packet ' $k$ ' at the receiver side respectively. $S_{k}$ is the packet size of the video layer.

The incorporation of the SP-driven admission control mechanism allows controlling the admission of the new traffic flows over network links. SP allocates e2e aggregated pipes for these flows such that each packet sent by a particular peer must not exceed the peak data rate. Hence, each packet sent must obey the rule: $\forall i: S_{i} / t_{i} \leq P D R$, where $t_{i}$ represents a time interval between two packets.

We can derive the following rule (Eq. 1) that allows the traffic test conformance (i.e. maximum throughput constraint): 


$$
\sum_{k=1}^{m} S_{k} \leq\left(\sum_{k=1}^{m} P_{k}^{\text {transmit }}\right) P D R
$$

The second constraint (Eq.2) is related to the draining of buffer at the receiver side, since we should be sure those packets still arrive at the destination.

$$
0 \leq \sum_{k=1}^{m} s_{k}-A D R \sum_{k=1}^{m} P_{k}^{\text {transmit }} \leq P B S
$$

The third constraint is related to the end-to-end delay. Eq. 3 represents e2e delay of the arrival video packets from sender to receiver. This constraint is more important as the real-time applications require minimum delay and play a major role in the overall QoS. Packet loss occurs when the deadline delay is passed.

$$
0 \leq \sum_{k=1}^{m} P_{k}^{\text {receive }}-\sum_{k=1}^{m} P_{k}^{\text {transmit }} \leq D_{\max }
$$

In Eq. 3, $D_{\max }$ can be specified on the basis of estimated maximum RTT that can be tolerated between the sender and the receiver node. However, in streaming applications, we may tolerate some delay for the draining buffer (10s-15s). In our proposal, $D_{\max }$ is inspired from the retransmission timer technique used in TCP.

$$
D_{\max }=\min \left[U_{\text {bound }}, \max \left[L_{\text {bound }},(\alpha * S R T T)\right]\right]
$$

In Eq. $4, U_{\text {bound }}$ represents the maximum size the receiver side buffer, and $L_{\text {bound }}$ represents the minimum bound for buffer that can be $50 \%$ of the $U_{\text {bound }}, \alpha$ is factor of variance of delay, and SRTT corresponds the smooth RTT estimates by an exponential average that gives more weight to the recent RTT estimates to the older estimates.

\section{Performance Evaluation}

We evaluated the proposed mechanism using ns-2 simulator [13]. We used the SVC based video encoding for the MPEG-4 video trace file "Simpsons" [14] into four different video layers, i.e. base layer offering $40 \%$ of original video quality, enhancement layer 1, 2, and 3 offering $25 \%, 20 \%$ and $15 \%$ of the video quality respectively. We distributed SVC based video layers among different sender peers to examine the performance of the proposed admission control based QoS mechanism. The video traces used for simulations provide VBR (variable bit rate) video stream and SP allocates the network resources on the basis of requested video layers. We performed simulations over the network topology presented in [12]for two scenarios as follows:

- Scenario without Admission Control based Quality Adaptation: In this case, P2P system works like downloading mode without any admission control mechanism.

- Scenario with Quality Adaptation: In this case, QoS provision mechanism is implemented by incorporating admission control where SP allocates the end-to-end bandwidth for the content delivery among sender and receiver peers as described in section 3 .

We focus on many-to-one streaming scenario where a single receiver (CC) intends to receive the real-time video packets from multiple sender peers (CPs). To address the dynamicity of P2P networks, we implemented a peer/stream switching mechanism as presented in [12]. The CC requests the video file directly from the service provider. SP has a global view of network condition with explicit communication with network providers and maintains a traffic forecast matrix for the on-going communications and available bandwidth over certain paths. The communication among SPs and NPs are carried by SLA/SLS negotiations. For the scenario with admission control based quality adaptation, the actual content delivery is carried out by allocation of e2e aggregate pipes on the basis of TSPEC (traffic specification) and no other traffic is allowed to pass through these dedicated pipes. These TSPEC parameters include the peak data rate, average data rates and inter packet arrival delay for the each video quality layer while satisfying the constraints (Eq. 1, Eq. 2, and Eq. 3) present in section III. These constraints allow the admission control mechanism to admit new traffic flow over certain links. Fig. 3 presents the received video throughput in both scenarios along with the expected video quality of the original video. Fig. 4 presents the packet drops ratio for the received video at receiver-end. We notice huge packets loss ratio in the scenario without applying any admission control based QoS mechanism.

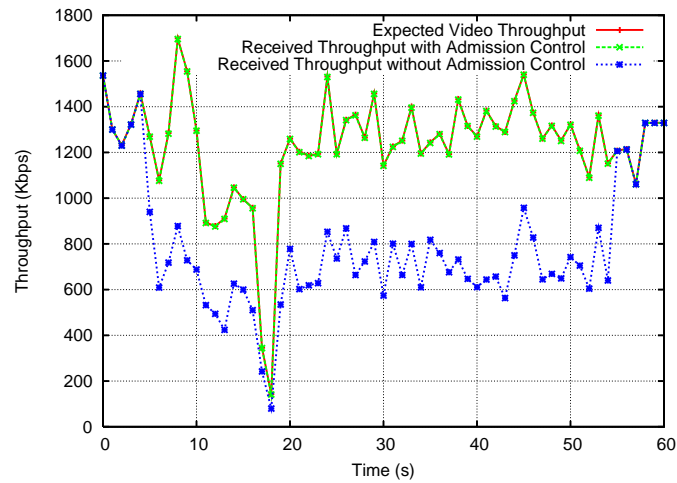

Fig. 3. Received Video Throughput

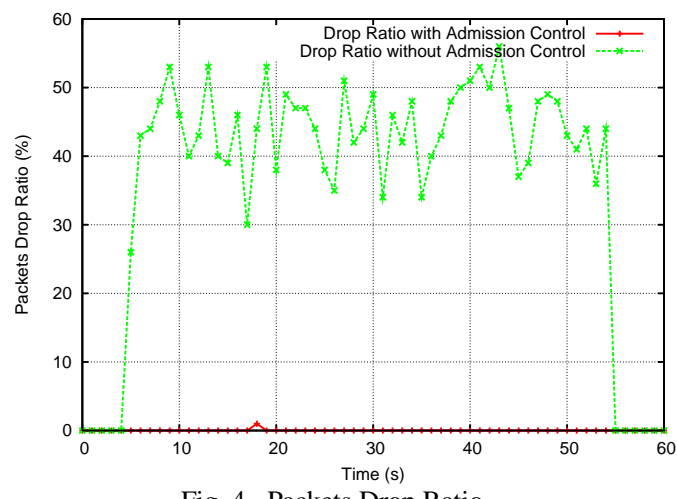

Fig. 4. Packets Drop Ratio

These results give a brief picture of the SP-driven traffic forecast matrix that is used for the installation of the QoS enabled aggregate pipes over the e2e links. The traffic descriptor-based admission control mechanism enables the QoS provision for the streaming application but still it requires more analysis to determine its performance for other parameters SLA/SLS parameters.

We notice a clear difference between the received video qualities that is almost $100 \%$ when streaming mechanism is supported by proposed admission control mechanism, while in 
the other scenario video content are delivered over shared network paths. The other traffic passing over the same networking link resulted into huge packet drop ratio and affected the received video quality drastically that is not acceptable for real-time content delivery. We noticed that enhancement video layer 3 is receiver $100 \%$ even in the scenario when we did not apply any admission control mechanism, but it can not contribute to enhance the received video quality when the base video layers not received completely.

Fig. 5, Fig. 6 and Fig. 7 present the comparison between received and original base layers, enhancement layer 1 , enhanced layer 2, and enhanced layer 3 respectively. We can see clearly that our proposed mechanism performs a smooth video delivery with higher quality, lower loss that improves the overall QoS for the received video. The enhanced packets throughput and lower packet drop ratio result into enhanced received QoS and justify the viability of proposed admission control mechanism.

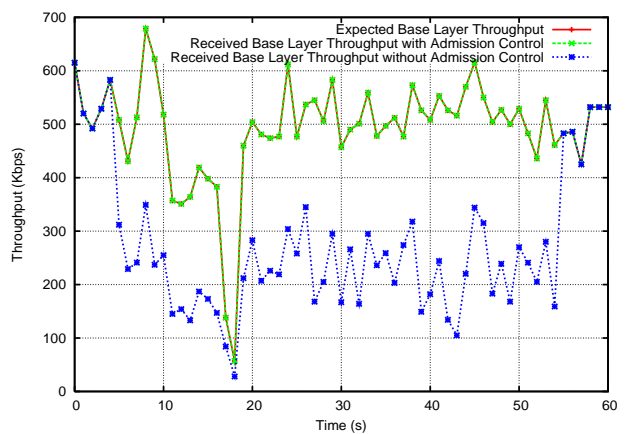

Fig. 5. Received Video Throughput for Base Layer

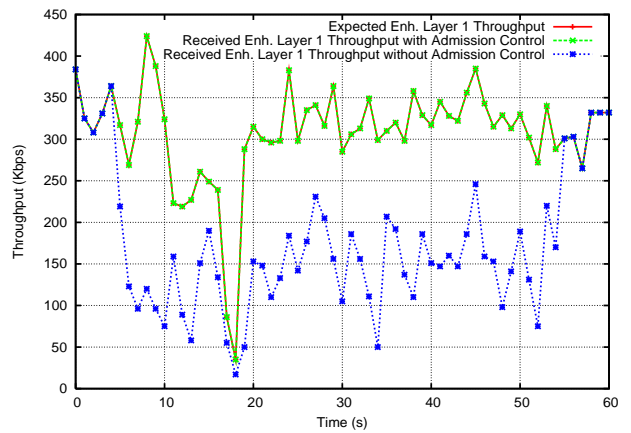

Fig. 6. Received Video Throughput for Enhancement Layer 1

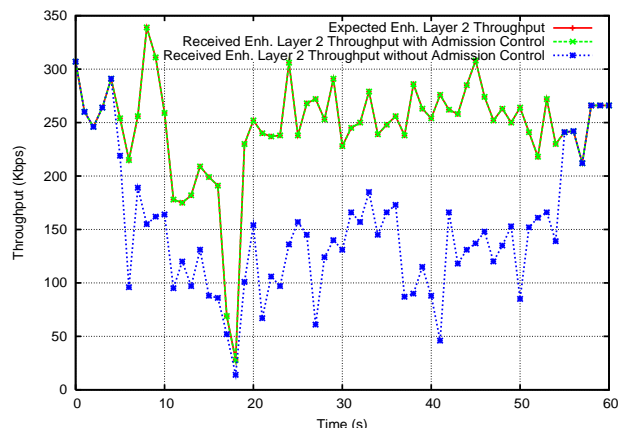

Fig. 7. Received Video Throughput for Enhancement Layer 2

\section{Conclusion \& Future Perspectives}

In this paper, we presented an admission control based QoS provision mechanism for the real-time video streaming over SP-driven P2P environment. The SP-driven approach makes our proposed mechanism distinguished to the other available solutions and provides efficient utilization of network resources. We observe a significant improvement in QoS for the received video in the scenario when our QoS provision mechanism is applied. In future, we aim to evaluate the proposed mechanism for large scale networks and to extend the mechanism for the efficient cooperation among different SPs for IPTV service delivery.

\section{REFERENCES}

[1] Light Reading, “Controlling P2P Traffic," available at http://www.lightreading.com/document.asp?d=lightreading\&doc _id=44435\&page_number=3 (last view: June 20, 2008)

[2] V. Aggarwal, A. Feldmann, and C. Scheidele, "Can ISPs and P2P Users Cooperate for Improved Performance?”, in proc. Of ACM SIGCOMM Computer Communication Review. Volume 37 , Issue 3 (July 2007)

[3] E. Marocco and V. Gurbani, "Application-Layer Traffic Optimization (ALTO) Problem Statement”, Internet-Draft, draftmarocco-alto-problem-statement-02, July 2008.

[4] H. Xie, A. Krishnamurthy, A. Silberschatz, and Y.R. Yang., "P4P: Explicit Communication for Cooperative Control Between P2P and Network Providers”, in P4PWG Whitepaper (2007). Available from http://www.dcia.info/documents/P4P_Overview.pdf

[5] E. Borcoci, G. Kormentzas, A.H. Asgari and T. Ahmed, "Service invocation admission control algorithm for multidomain IP environments” in Computer Networks. Volume 51, Issue 16, Pages 4669-4678 (2007)

[6] S. Georgoulas, P. Trimintzios, G. Pavlou, K.H. Ho, "Heterogeneous real-time traffic admission control in differentiated services domains", in proc. of IEEE Globecom 2005.

[7] S. Shenker and J. Wroclawski, "Network Element Service Specification Template”, IETF Draft. (1997) Online: Accesses 20 April 2008 from www.ietf.org/rfc/rfc2211.txt

[8] J. Reichel, H. Schwarz, and M. Wien. Joint scalable video model JSVM-8. Technical Report JVT-U202, Joint Video Team, Hangzhou, China, October 2006.

[9] K. Eger, and U. Killat, "Fair resource allocation in peer-to-peer networks (extended version)", in proc. of Computer Communications: Special Issue: Advances in Communication Networking, Elsevier, pp. 3046-3054, vol. 30, 2007.

[10] S. Son, “A Hybrid Peer-to-Peer Media Streaming”, in proc. of Grid and Cooperative Computing - GCC 2005. LNCS. vol. 3795, pp. 825-835. Springer, Heidelberg. 2005.

[11] C. Loeser, M. Ditze, and P. Altenbernd, "Architecture of an intelligent quality-of-service aware peer-to-peer multimedia network", in proc. of the 7th World of Multi conference on Systemics, Cybernetics and Informatics, July 2003.

[12] M. Mushtaq and T. Ahmed, "Hybrid Overlay Networks Management for Real-Time Multimedia Streaming over P2P Networks”, in proc. MMNS 2007, LNCS volume 4787, pp. 113, 2007.

[13] The Network Simulator (NS-2), http://www.isi.edu/nsam/ns/

[14] MPEG-4 Trace Files, available from http://www.tkn.tuberlin.de/research/trace/ltvt.html 